\title{
A Solution Approach for a Class of Parametric Linear Programming Problems

\author{
Mustafa SIVRİ ${ }^{1}$, İnci ALBAYRAK ${ }^{1}$, Kadriye ŞİMŞEK ALAN ${ }^{1 *}$, Gizem TEMELCAN $^{2}$
}

\begin{abstract}
Depending on the nature, objectives, and constraints of the decision variables; linear programming, nonlinear programming, integer programming, mixed integer programming etc. can be classified. Extensive research has been conducted to solve all types of these problems in a parametric context. In this paper, to solve optimization problems having uncertainties represented by a single parameter on the objective function, a systematic linearization approach is developed considering the parametric expression as nonlinear. In the proposed approach, the objective function is considered as nonlinear which is converted into linear by using first order Taylor series expansion at the points making the parametric costs zero. Thus, the optimal solution is obtained from the constructed linear programming problem. In this way, by determining the intervals in which the optimal solution changes, the solution of the parametric linear programming problem is obtained. A numerical experiment is illustrated to present the effectiveness of the proposed approach.
\end{abstract}

Keywords: Parametric linear programming, linearization technique, Taylor series expansion

${ }^{1}$ Mustafa SİVRİ (Orcid ID: 0000-0002-0524-8502), İnci ALBAYRAK (Orcid ID: 0000-0001-6906-9880), Kadriye ŞİMŞEK ALAN (Orcid ID: 0000-0001-6751-8013), Yıldız Teknik Üniversitesi, Kimya Metalürji Fakültesi, Matematik Mühendisliği Bölümü, İstanbul, Türkiye

${ }^{2}$ Gizem TEMELCAN (Orcid ID: 0000-0002-1885-0674), İstanbul Aydın Üniversitesi, Anadolu Bil Meslek Yüksek Okulu, Bilgisayar Teknolojileri Programı, İstanbul, Türkiye

*Sorumlu Yazar/Corresponding Author: Kadriye ŞİMŞEK ALAN, e-mail: ksimsek@yildiz.edu.tr 
A Solution Approach for a Class of Parametric Linear Programming Problems

\section{INTRODUCTION}

Linear programming (LP) can be defined as a mathematical method of allocating scarce resources to highly competitive activities when the problem can be expressed by using a linear objective function and linear inequality constraints. Although, more complex real-world problems cannot be expressed perfectly in terms of a set of linear functions, realistic representations of many real-world problems can be provided reasonably. Thus, the LP model should be defined that any changes caused by internal and external factors can be included. Therefore, the parameter appearing in the model that is neither known nor stable in the real-world is considered to be a variable.

Many of real-world problems represented by LP are formulated as deterministic optimization problems in which the coefficients of objective function and constraints are constants such as demands, availabilities, prices, resource requirements, etc. Coefficients can be taken as parameters in LP problems, which take into account the variation of coefficients called parametric linear programming (PLP) problems. PLP is used systematically to analyze precision by gradually changing the model parameter. Uncertainties caused by the uncontrollable parameter can be classified as objective function (OF), left-side of restrictions (LHS) or right-side of restrictions (RHS). For example, when it is allowed to change OF coefficients, costs in optimization problems, the solution behavior is asked to be examined. As an extension of the sensitivity analysis of PLP, it investigates changes in the optimum LP solution with variations of OF, RHS and LHS. Sensitivity analysis is a technique in the field of optimization and is used to determine how different values of an independent variable affect a dependent variable under several assumptions. PLP has many applications in waste management, fleet planning, model estimation control, process synthesis, scheduling problems, and production programs. Various researches have been conducted in this field that provide applicable solutions for parametric mixed integer LP, interval LP and parametric LP.

Recent articles have considered PLP with parameters on OF or RHS, but there are a few studies where parameters are on LHS of the constraints.

Gass and Saaty (1955a) studied the parametrization of cost function in the general LP problem with one parameter, and then they (1955b) generalized it to the n-parameter case. Adler and Monteiro (1992) studied parametric-RHS LP problem and defined a new optimality interval consisting of either a breakpointor an open interval between two successive breakpoints of the continuous piecewise linear convex function. Cambini et al. (1993) considered parametric linear fractional programming with an unbounded feasible region. They proposed solution procedures based on modification of Martos' algorithm or Charnes-Cooper's algorithm and applied each of these procedures to the problems having parameter either on the OF or on RHS. Rujun and Xinyuan (2012) extended the traditional simplex based parametric programming methodology by adjusting OF coefficient parameters of the large LP problem. Simplex method developed by Dantzig (1963) was first discussed by Gass and Saaty (1955a) to solve the parametric RHS LP problems. PLP problem in which the coefficient matrix is parameterized has been studied by Saaty (1959), Courtillot (1962), Willner (1967) and Barnett (1968).

A class of parametric programming problems which can be used such as in planning and scheduling techniques under uncertainty is discussed. Parametric programming methods determine the effect of the parameter on the optimal solution if the LP problem contains a parameter. In point of view, the aim of this study is to propose a solution methodology for the situations in which the coefficients of decision variables in OF is a parameter. Linearization, as a remarkable approach based on Taylor series expansion, is utilized to find a solution for a kind of PLP problems.

This paper is organized as follows: Section 2 presents required information. In Section 3, the proposed approach is handled. Section 4 and Section 5 consist of our numerical example and conclusion, respectively.

\section{PRELIMINARIES}

In this section, required information and definitions of the PLP problems with a parameter in OF will be presented. 
Definition 2.1. A general constrained LP problem can be defined as follows:

$\min f(x)$

s.t.

$g_{e}(x)=0, e=1,2, \ldots, \ell$

$g_{i}(x) \leq 0, i=\ell+1, \ldots, m$

where $x=\left[x_{1}, \ldots, x_{n}\right] \in R^{n}$ is a vector, $f: R^{n} \rightarrow R, g_{e}: R^{n} \rightarrow R$ and $g_{i}: R^{n} \rightarrow R$ are linear functions and $m \leq n$

Definition 2.2 (Chong and Zak, 2013). Any point $x$ satisfying the constraints is called feasible point. The set of all feasible points is called a feasible set, i.e. $X=\left\{x \in R^{n}: g_{e}(x)=0, e=1, \ldots, l ; g_{i}(x) \leq 0, i=l+1, \ldots, l+m\right\}$.

Definition 2.3. A feasible solution $x^{*}$ as the smallest objective function value is called an optimal solution of a minimization problem in LP.

Definition 2.4. PLP problem in standard form with a single parameter in OF is

$\operatorname{Min} Z(\theta ; x)=\sum_{j=1}^{n}\left(c_{j}+\alpha_{j} \theta\right) x_{j}$

s.t.

$\sum_{j=1}^{n} a_{i j} x_{j} \leq b_{i}, \quad i=1,2, \ldots, m$

and

$x_{j} \geq 0, \quad j=1,2, \ldots, n$

where $\theta$ is a parameter and each $\alpha_{j}$ is a given input constant indicating the relative rates at which the coefficients will be changed.

\section{PROPOSED APPROACH}

A linearization method based on Taylor series expansion is adopted to solve the PLP problem (1) that is called parametric cost problem (PCP). Namely, OF having a parameter is converted into linear form by using the linear terms of Taylor series expansion at the points which makes the parametric costs zero. By considering these specified points, the range of parameter is determined such that the optimal solution remains invariant.

The OF of the PLP is considered as $f\left(x_{1}, x_{2}, \ldots, x_{n}\right)$, and $A=\left(a_{1}, a_{2}, \ldots, a_{n}\right)$ is a point such that $f\left(a_{1}, a_{2}, \ldots, a_{n}\right)=0$. Recall that, Taylor series expansion of $f(x)$ of a single variable around the point $a$ is given in (Remani, 2013) by

$f(x)=f(a)+f^{\prime}(a)(x-a)+\frac{1}{2 !} f^{\prime \prime}(a)(x-a)^{2}+\ldots+\frac{1}{n !} f^{(n)}(a)(x-a)^{(n)}+\ldots$

namely,

$f(x)=f(a)+f^{\prime}(a)(x-a)+$ higher order terms.

For $x$ sufficiently close to $a$, higher order terms will be close to zero and are dropped to obtain the following approximation 
$f(x) \approx f(a)+f^{\prime}(a)(x-a)$.

By extension, each $f$ in $n$ variables can be linearized as

$f(x) \approx f(A)+\frac{\partial f(A)}{\partial x_{1}}\left(x_{1}-a_{1}\right)+\frac{\partial f(A)}{\partial x_{2}}\left(x_{2}-a_{2}\right)+\ldots+\frac{\partial f(A)}{\partial x_{n}}\left(x_{n}-a_{n}\right)+\ldots$

The generated approach to solve PLP is presented below.

\section{The Proposed Algorithm}

The proposed algorithm generates a solution based on systematic changes in OF coefficients. The presented algorithm can be applied by making the parametric costs, i.e. the objective function coefficients, zero. Thus, the intervals of parameter are determined such that the current basis will stay optimal in the specified interval.

Step 1: Load PLP problem containing a single parameter in the OF.

Step 2: Examine the coefficients of OF, specify the parameter values $t^{*}$ in the real axis such that the parametric costs zero and construct the intervals in the nonnegative real axis.

Step 3: Solve the constructed LP problems at each $t^{*}$.

Step 4: Expand the OF to Taylor series at the optimal solution obtained for each $t^{*}$ in Step 3.

Step 5: For each $t^{*}$, consider $t \leq t^{*}$ as a new constraint and solve the constructed LP problem. Because the solution of LP problem will be unbounded for $t \geq t^{*}$, the obtained solution will be valid from the RHS of $t^{*}$ to the following parameter value. The obtained solution will be invariant in the RHS of the last parameter value in the real axis.

Step 6: Specify the intervals of the parameter relative to the changing basis.

Step 7: Determine the optimal solution as a function of parameter.

The proposed analytical approach can be presented as a modification of the simplex method for solving PLP problems having single parameter. Thus, we can determine when and how the optimal solution changes to handle parametric analysis of that kind of problems in which the costs of $\mathrm{OF}$ are represented by parameter.

\section{NUMERICAL EXPERIMENT}

Consider the following PLP problem (Taha, 2007):

$$
\begin{aligned}
& \max z(t, x)=(3-6 t) x_{1}+(2-2 t) x_{2}+(5+5 t) x_{3} \\
& \text { s.t. } \\
& x_{1}+2 x_{2}+x_{3} \leq 40 \\
& 3 x_{1}+2 x_{3} \leq 60 \\
& x_{1}+4 x_{2} \leq 30 \\
& t \geq 0 \text { and } x_{1}, x_{2}, x_{3} \geq 0 .
\end{aligned}
$$

Steps 1-2: The costs of OF are examined and $t^{*}=\{-1,0.5,1\}$ are determined. Because of $t \geq 0, t^{*}=-1$ is not considered. 
Step 3: The constructed LP problems is solved at each $t^{*}$.

For $t^{*}=0$, the LP problem is

$\max z=3 x_{1}+2 x_{2}+5 x_{3}$

s.t.

$x_{1}+2 x_{2}+x_{3} \leq 40$

$3 x_{1}+2 x_{3} \leq 60$

$x_{1}+4 x_{2} \leq 30$

and the optimal solution is $\left(x_{1}, x_{2}, x_{3}\right)=(0,5,30)$. Similarly, while $t^{*}=0.5$ and $t^{*}=1$, the optimal solutions are $(0,5,30)$ and $(0,0,30)$, respectively.

Steps 4-5: By determining $\frac{\partial z}{\partial x_{1}}=3-6 t, \frac{\partial z}{\partial x_{2}}=2-2 t, \frac{\partial z}{\partial x_{3}}=5+5 t$ and $\frac{\partial z}{\partial t}=6 x_{1}-2 x_{2}+5 x_{3}$, (2) is used to expand the objective function to Taylor series for each obtained optimal solution at each $t^{*}$. The expansions of OF corresponding to each parameter value is presented in Table 1.

Table 1. The expansions of OF corresponding to each parameter

\begin{tabular}{ccl}
\hline Parameter values $t^{*}$ & The obtained optimal solution & \multicolumn{1}{c}{ Objective function } \\
\hline$t^{*}=0$ & $\left(x_{1}, x_{2}, x_{3}\right)=(0,5,30)$ & $z_{1}^{*}=3 x_{1}+2 x_{2}+5 x_{3}+140 t$ \\
$t^{*}=0.5$ & $\left(x_{1}, x_{2}, x_{3}\right)=(0,5,30)$ & $z_{2}^{*}=x_{2}+7.5 x_{3}+140 t$ \\
$t^{*}=1$ & $\left(x_{1}, x_{2}, x_{3}\right)=(0,0,30)$ & $z_{3}^{*}=-3 x_{1}+10 x_{3}+150 t$
\end{tabular}

For $t^{*}=0$, the following LP problem is solved

$\max z_{1}^{*}=3 x_{1}+2 x_{2}+5 x_{3}+140 t$

s.t.

$x_{1}+2 x_{2}+x_{3} \leq 40$

$3 x_{1}+2 x_{3} \leq 60$

$x_{1}+4 x_{2} \leq 30$

$t \leq 0$

and the basis is found as $\left(x_{2}, x_{3}, x_{7}\right)=(5,30,10)$. Similarly, the bases are found as $\left(x_{2}, x_{3}, x_{7}\right)=(5,30,10)$ and $\left(x_{3}, x_{5}, x_{7}\right)=(30,10,30)$ corresponding to $t^{*}=0.5$ and $t^{*}=1$, respectively.

Step 6-7: Specify the interval of the parameter respect to the changing basis and determine the optimal solution as a function of parameter in the following Table 2 .

Table 2. The interval of the parameter respect to the changing basis

\begin{tabular}{ccccc}
\hline Parameter interval & $x_{1}$ & $x_{2}$ & $x_{3}$ & $z$ \\
\hline $0 \leq t<1$ & 0 & 5 & 30 & $160+140 t$ \\
$t \geq 1$ & 0 & 0 & 30 & $150+150 t$ \\
\hline
\end{tabular}




\section{CONCLUSION}

PLP is a kind of problem to investigate the effects of changes on parameter in the OF. The usage of PLP adds a new dimension to LP that consider the parametrized factor. PLP is an extension for systematic sensitivity analysis which is especially valuable for post-optimality analysis. In real applications, parameters have some degree of uncertainty, and sensitivity analysis must be conducted. From this point of view, linearization has been performed using first order Taylor polynomial and a solution approach has been developed. The proposed approach has a remarkable advantage to find acceptable solution for decision makers in terms of ease of application and accuracy of results. The main advantage of the considered parametric analysis problem is the investigation of trade-offs in parameter value.

\section{REFERENCES}

Adler, I. \& Monteiro, R. D. (1992). A geometric view of parametric linear programming. Algorithmica, 8(1-6), 161-176.

Barnett, S. (1968). A simple class of parametric linear programming problems. Operations Research, 16(6), 1160-1165.

Cambini, A., Schaible, S. \& Sodini, C. (1993). Parametric linear fractional programming for an unbounded feasible region. Journal of global optimization, 3(2), 157-169.

Chong, E. K. \& Zak, S. H. (2013). An Introduction to Optimization (Vol. 76). John Wiley\&Sons.

Courtillot, M. (1962). On varying all the parameters in a linear-programming problem and sequential solution of a linear-programming problem. Operations Research, 10(4).

Dantzig, G.B. (1963). Linear Programming and Extensions, Princeton University Press, Princeton, NJ.

Gass, S. \& Saaty, T. (1955a). The computational algorithm for the parametric objective function. Naval research logistics quarterly, 2(1-2), 39-45.

Gass, S. I. \& Saaty, T. L. (1955b). Parametric objective function (part 2)-generalization. Journal of the Operations Research Society of America, 3(4), 395-401.

Huang, R. \& Lou, X. (2012). A Simplex Based Parametric Programming Method for the Large Linear Programming Problem. In Proceeding of the International Multiconference of Engineers and computer scientists (Vol. 2, pp. 14-16).

Remani, C. (2013). Numerical methods for solving systems of nonlinear equations. Lakehead University Thunder Bay, Ontario, Canada.

Saaty, T. L. (1959). Coefficient perturbation of a constrained extremum. Operations Research, 7(3), 294302.

Taha, H. A. (2007). Operational Research: An Introduction. Pearson/Prentice Hall, 8th edition.

Willner, L. B. “On Parametric Linear Programming,” SIAM J. Appl. Math., Vol. 15, No. 5 (Sept., 1967), pp. 1253-1257. 